\title{
Protocol and beyond: practices of care during a Tuberculosis vaccine clinical trial in South Africa
}

\author{
Justin Dixon \\ Social Anthropology, University of Cape Town, Private Bag X3, Rondebosch 770I, South Africa \\ dxnjus00I@myuct.ac.za
}

\begin{abstract}
Much of the current social science literature on the clinical trials industry focuses on the profit-seeking practices adopted by pharmaceutical companies and the contract research organisations they employ to enable the mass production and distribution of their products. However, what the current literature demands is further ethnographic engagement with the particularities of the diseases being investigated, the local contexts and histories in which they are entwined, and how these impact the affective relationships between clinical research organisations and their participants. On the basis of ethnographic research with a nonprofit clinical research organisation specialising in TB vaccinations in South Africa, I argue that the complexities of TB mean that research into it necessitates frequent and often intimate interactions with research participants. These were perceived by researchers to yield opportunities to take an interest in the physical and psychosocial wellbeing of research participants which went beyond and sometimes ran into conflict with the requirements of protocol. The aim of this paper is to advocate more finely tuned attention to the challenges posed by the clinical trials industry today, an attention sensitive to the particularities of the contexts of clinical trials.
\end{abstract}

Key Words: Clinical trials, tuberculosis, care, experiment, protocol, ethics, South Africa

\section{Introduction}

The clinical trials industry, having emerged in response to a global 'need' for data procured from human bodies, has recently attracted the attention of a number of social scientists. The primary focus of this increasing body of literature is the exploitative practices adopted by 'Big Pharma' in the pursuit of profit and, in particular, the ways in which it is able to enlist the bodies of impoverished people and populations worldwide to this end (Adams et al. 2005; Fischer 2009; Petryna 2007, 2009). In this paper, I argue that whilst these critiques are highly important, further ethnographic attention is required to the particularities of the diseases at which much clinical research is directed, the local contexts and histories in which they are entwined, and how these impact the affective relationships between clinical research organisations and their participants. My contention, one which is recommended by current literature on practices of care (e.g. Mol 2008), is that these are important to consider if we as critical enquirers are to understand the clinical trials industry and the challenges it currently faces, especially as more and more clinical trials are being relocated to resource-poor regions of the world.

After a brief elaboration on the relevant literature below, in order to substantiate these claims I draw upon ethnographic research conducted with the Tuberculosis Research Organisation (TRO), a non-profit research organisation in South Africa specialising in clinical trials on tuberculosis (TB) vaccines. Detailing some of TRO's day-to-day operations, I argue that conducting clinical research on TB in South Africa - and childhood TB in particular - necessitates frequent and often intimate interactions with often incredibly impoverished research participants (in the context of the 'follow-up visits' and the 'case verification ward'). These interactions were regarded by TRO's researchers as yielding opportunities to take an active interest in the physical and psychosocial wellbeing of their research subjects which often went beyond the requirements of protocol (cf. Human 20I I). As I shall demonstrate, some of the most pressing challenges TRO's researchers faced as they tried to conduct clinical research in the midst of often great suffering pertained to how to approach the 'extra-protocol' provision of care for research participants. But these challenges cannot easily be abstracted from the particularities of TB as a historical phenomenon in South Africa, or from the legacy of apartheid governance which has left the majority of TRO's participants in nearabsolute poverty. The importance of context in the case of TRO suggests that the trajectory of the literature on clinical trials should be broadened to include more 'thick' ethnographic accounts, ones which will hopefully let us suggest some ground-level solutions to particular challenges in particular localities.

\section{Background}

The clinical trials industry brings together in an uneasy relationship the global neo-liberal economy, international pharmaceutical companies and regulatory bodies, independent research organisations, and low-income people/populations (Adams et al. 2005; Fischer 2009; Petryna 2007, 2009). The commodity that lies at the heart of this thriving industry is data procured from human subjects, which, when converted

I. The author would like to thank: two anonymous reviewers for their insightful comments, Helen MacDonald for going through drafts of this paper; UCT Interim Funding for making this research possible; and, most of all, The Tuberculosis Research Organisation for their ongoing co-operation and support. 
into 'go-aheads' from global regulatory bodies (e.g. the US Food and Drug Administration [FDA]) to mass produce and distribute prospective pharmaceutical bio-products, are worth millions or even billions of dollars in profit (Rennie 2010:114). Thus it is unsurprising that, as with numerous other 'natural' resources, people compete with one another for a share of the profits. One of the major structural readjustments to the clinical trials industry in the last three decades is pharmaceutical companies' outsourcing their clinical trials to independent, contract-based research organisations (CROs). Competition among these companies to secure contracts is fierce because their survival depends on the ability to get the job done efficiently and cheaply. Given that the most 'ideal' bodies for clinical research - ones which are relatively 'treatment-naive' - are to be found in resource-poor environments, a staggering number of clinical trials contracts have been awarded to research organisations geographically outside the industrialised centres of science (Adams et al. 2008:317).

On the one hand, participants in clinical trials are usually paid for their participation and, moreover, often enjoy access to healthcare resources they would otherwise be denied (Benatar 2002:II35). On the other hand, as authors have observed, these considerations, and efforts to adapt the neoliberal central pillars of ethical research - e.g. informed consent, protection from harm, minimising risk, confidentiality to the socio-cultural particularities of clinical research settings, are marked by the gaping structural inequalities that exist between researchers and vulnerable people and populations (Adams et al. 2005:268; Fischer 2009; Petryna 2009; Benatar and Upshur 2010). Indeed, as Petryna (2007:294) astutely notes, 'Big Pharma' is now powerful enough to manipulate the global variability in regulatory standards to fill their clinical trials, and ultimately get their products on the market. Seen against the backdrop of such over-arching profit-seeking practices, it is perhaps unsurprising that anything more than a face-value ethic of care for research subjects seems to be, although not altogether absent (see, for instance, Fischer 2009), at least considerably undernourished. These are important concerns, and they are yet to be given the attention they merit in biomedical forums with a focus on the global reach of contemporary clinical trials.

Yet, recent anthropological scholarship on practices of care suggests that this expanding body of literature on the clinical trials industry would be complemented by further ethnographic attention to particular localities of clinical research. Where the latter body of literature has drawn attention to the neo-liberal vocabulary legitimising the global conduct of clinical trials, the former, along a similar theoretical vein, has challenged the "logic of choice" as the paradigm for formal healthcare provision (Mol 2006, 2008; Mol, Moser and Pols 2010; Henderson 20I I; Hardon et al. 20I I). Contra the "logic of choice" paradigm, Annemarie Mol (2008) importantly argues that healthcare is not the product of 'rational', 'informed' choices made on the part of individual patients nor does it have a clear-cut beginning and end. She demonstrates that in reality, care-provision involves on-going, obstacle-ridden processes of negotiation and "tinkering" (Mol 2008:14) between multiple actors, including patients, their families, medical professionals, and - since Mol is influenced by actor- network theory (e.g. Latour 1999) - various medical tools, technologies and procedures. Care is a relational, processual phenomenon. Mol (2008:9) argues that the particularities of these processes are context-specific, inevitably varying from location to location. Therefore, creating productive and efficacious solutions to the challenges of providing 'good' care requires close attention to the local ecologies of care which enable, and inhibit, particular initiatives.

The affective relations between clinical researchers and their participants are undeniably marked by the wider political-economic processes bringing them into frequent contact. Nonetheless, the above considerations recommend further ethnographic engagement with the particularities of context both human and non-human - informing the challenges faced by clinical researchers on the ground as they try to balance, on the one hand, the arguably inherent exploitation entailed by clinical trials, and, on the other, the subjectivities and healthcare needs of impoverished research participants. In order to address this gap, I turn the reader's attention to the current state of the tuberculosis (TB) epidemic in South Africa and, subsequently, to the endeavours of the TRO as they conducted a clinical trial designed to test a prospective TB vaccine on the bodies of infants living in a rural region of the country.

\section{TB vaccinations research at TRO}

Tuberculosis (TB) is re-emerging as one of the most prevalent infectious diseases in the world. In 2007, I.3 million were killed by TB alone and an additional 456,000 were killed who were already co-infected with HIV/AIDS (World Health Organisation 2009). The vast majority of positive TB diagnoses are made in low-income countries, where the conditions under which the disease thrives - inadequate food security, unhygienic and crowded living conditions, and poor access to healthcare - are abundant (Benatar and Upshur 2010:1215). Despite being a democratic republic since 1994, a middle-income country and having a large number of people in high-income brackets compared to low-income countries, South Africa remains haunted by a legacy of apartheid governance that left millions in almost absolute poverty. Thus, in conjunction with an already critical epidemic of HIV and AIDS, which compounds the effects of TB, it is perhaps unsurprising that certain provinces in the country have amongst the highest TB morbidity and mortality rates in the world (Naidoo and Mwaba 2010:1324). To make matters worse, although it was once thought that Africa had a comparatively low rate of drug resistant strains of TB compared to other continents, the high incidence of patients 'defaulting' on their drug-therapies has ensured outbreaks of several multiple-drug-resistant strains of TB (Wallengren et al. 20l I).

Notwithstanding pharmaceutical companies' comparatively low level of interest in TB, significant time and resources are being concentrated on managing the disease worldwide. This has involved the development not only of post-diagnostic antibiotics - which are unfortunately becoming less and less effective given the high incidence of drugresistant $T B$ - but also research into vaccinations to preclude the onset of TB disease in the first instance. The Bacilli Calmette-Guerin (BCG) vaccine, which has been an integral part of the TB response since its introduction in the first half of the 
$20^{\text {th }}$ century, is currently the only vaccine on the market. However, it is widely thought to be insufficient as a self-standing TB preventative. Thus, a primary goal at present is the production and distribution of a supplementary booster vaccine which will increase the worldwide efficacy of the BCG.

TRO is currently trialling TB vaccines trials in South Africa in the interests of testing such a vaccine. TRO's endeavours necessitate a wide institutional base and a regular flow of organic matter between its main laboratory (located in a large city in South Africa) and its field-project office in a town about an hour's drive away. The location of the field project office where the trials are conducted was chosen because of its close proximity to the pool from which research participants are drawn, a several-hundred-thousand strong rural population. The field-project office - where I conducted most of my ethnographic research - is located within the premises of a public hospital which specialises in TB diagnosis and treatment. It incorporates a vaccine trials clinic, a case verification (CV) ward for suspected cases of childhood TB, an immunology laboratory, and various administrative offices. It also has upward of thirty vehicles for collecting organic matter from, and delivering it to, the laboratory in the nearby city, as well as picking up and dropping off the donors of that organic matter in and around local clinics in the surrounding rural region.

TRO's research is made incredibly difficult because of the complexities inherent in conceptualising and diagnosing TB, especially in the infants and young children on whom the majority of TB vaccines research is conducted. A great many signs and symptoms are associated with childhood TB: weight loss, a cough lasting more than two weeks, certain blotches on a chest X-Ray, positive quantiFERON or Mantoux skin test (both of which test for latent TB), and positive 'TB culture'. And an infant may, as far as anybody can actually know, have TB disease, and yet display only some of these symptoms and signs. Consequentially, diagnostic "algorithms" - tailored to context (Hatherill et al. 2009) - are used in the research context. These take as inputs various clinical, radiological and immunological signs and symptoms and give as outputs either positive or negative diagnoses of TB disease. More specifically, as a criterion an infant may be required to display, say, at least one of each type of sign or symptom - radiological, immunological, and clinical - to be diagnosed as having TB disease. In this way, researchers can be certain that there is a very high likelihood that a particular infant, despite not displaying various other signs or symptoms, has TB disease.

Sponsored by a large US-based non-profit organisation, the TRO is currently conducting (phase Ilb) clinical trials into the safety (and, as a secondary objective, efficacy) of a particular candidate booster vaccine for the BCG. ${ }^{2}$ This meant that not only are TRO's researchers vigilant for signs of TB disease in their research subjects, but also other 'adverse events' of varying degrees of severity that are causally linked to the trial vaccine. In this safety study, several thousand infants, enlisted from prenatal clinics around the rural communities, were vaccinated over a staggered time period of two years. After receiving the trial vaccine, each infant is to be 'followed up' regularly for a period of two years. Thus, the trial is scheduled to run for approximately four years. During that period, the infants' mothers - or relatives - are expected to make the infant available for several clinic and 'follow-up' visits, the purpose of which is to assess the infant's wellbeing and to conduct various tests and examinations. Aside from remaining vigilant for adverse events, if, during these visits, reason is found to suggest that an infant may have TB, the mother or relative is requested to attend the $C V$ ward on a prescribed date for a period of 48 hours, where the infant is subjected to a series of tests for childhood TB. Throughout the trial, drivers transport blood samples and other organic matter at least daily to and from TRO's urban laboratories, where the results of the tests are determined. The data collected by the home visit staff and clinics, as well as the results of the tests conducted in the CV ward, constitute the inputs into TRO's trial algorithm and, more generally speaking, the data which is ultimately used to determine whether the vaccine is safe for mass consumption.

\section{Following protocol and beyond: complexities on the ground}

While TB vaccinations research appears daunting even on paper, especially given the quantity and variety of clinical data required to make a diagnosis, in practice it is a colossal task. Completing it requires massive amounts of planning, a large number of staff, as well as abundant time and resources which, I was frequently told, TRO is hard-pushed to maintain. Moreover, as I became more acquainted with the goings-on in and around TRO's field office, I quickly came to realise that not only is the enactment of protocol on the ground rife with complexities, challenges, and contestation invisible from the abstract point of view of the protocol, it is also punctuated by the co-enactment - and the problems that arise therewith of what might be called 'extra-protocol'. For, in the midst of following the frequent, demanding, and often intimate requirements of protocol, the physical and psycho-social wellbeing of research participants (beyond that 'relevant' to the trial itself) has become an active, albeit contested and problematic, issue among TRO's on-site staff. The loci of these issues are the points at which there is the most direct social interaction between TRO's research staff and their participants: the 'follow-up' visits and admissions to the CV ward.

\section{Follow-up visits}

An integral part of TRO's research protocol is the 'follow-up' a.k.a. 'home' visits. As I mentioned before, the purpose of these visits, according to protocol, is to determine whether or not any of the enrolled infants are showing signs that they have TB disease or any other 'adverse events', in which case they need to attend the CV ward for further tests or treatment. This is no easy task. Given the large sample of participants required by TRO's phase Ilb trials - several thousand infants, in this case - and the staggered time period over which they have been given the trial vaccine, several teams of field researchers, co-ordinated by various administrative staff, head out into the surrounding region each day to track down a specified number of the enrolled infants. Each team has at least two members: a driver, who is responsible for finding

2. TRO is sponsored by several other institutions for different projects. 
the infants' homes, and another researcher, who conducts the home visit itself. A third team member is often included to make the process run more smoothly and efficiently.

One of the first things which caught my attention when I went out into the field with one of the home visit teams was how common a sight the TRO vehicles - white, with a memorable logo on the side - and research staff were to the people living in the settlements and farms around the field office. As we drove through one settlement, barely more than a passing glance was spared in our direction, and the few we received were mostly directed at me, suggesting that it was only because I was not a regular member of the team. When I asked Yolanda - one of the field researchers - about this, she said that while it took a significant amount of time before the constituents of the region were accustomed to TRO's presence, they are now, years later, such a familiar sight that they are now most frequently referred to as "the TB people". Perhaps as a consequence of this nurtured degree of familiarity, an air of routine permeated the interactions with infants' mothers or relatives as we made our way through the list of that day's infants. The infants' mothers or relatives, after meeting the TRO vehicle outside, were quick and, seemingly unhesitant, to allow the team into their homes and to make physical contact with the infants. Often, they were even willing to extend exchanged pleasantries into more personal chats regarding the ins-and-outs of their day-to-day lives. These exchanges suggested an intimacy between researchers and the areas in which they worked which I had not expected to see in the middle of what is effectively a bio-scientific experiment.

Nonetheless, all parties knew what the follow-up visit team's presence entailed (see above). Guiding this task is the follow-up visit form, a series of questions and boxes to be filled in, most of which are to be answered on the basis of the testimony of the infants' mothers or relatives. Questions include whether the infant has been in contact with anyone with TB recently, whether they have been coughing excessively, and, if so, for how long, and if they have had other medical problems or been taking any medicines since the last visit. There are also some pragmatic questions to be answered, such as how many attempts it took to track down the infant, the relation of the infant to the present carer, and a space for any further comments. Perhaps the most characteristic aspect of the visit, however, and by far the most difficult to accomplish, is the weighing of the infant. This is distressing for the infant because, in the interests of procuring a 'reliable' measurement, the infant needs to be stripped of their clothes - a necessity made even worse by the fact that it was winter at the time of my fieldwork. In one home, it took several attempts for Yolanda, Lucinda, and the mother to convince an increasingly distraught infant to stand up on TRO's pair of scales long enough to procure a reading. Even then, the infant would not stand still enough and so they had to add a bowl to the scales - one used to weigh younger infants who are unable to stand of their own accord - to get the desired measurement.

Asking the prescribed questions and weighing the infant is, in effect, all that the study protocol requires of the followup teams. However, having sat in on one of the weekly "TB Reviews" - a seminar, on this occasion conducted by Dr van
Zyl, which TRO's staff are strongly advised to attend - I was aware that the follow-up teams are also implored by the study doctors to take a holistic view of the infant during the home visit, one which often necessitates a critical eye for context that transcends the bare requirements of the form. This includes, for instance, whether they live close to a sewer, are malnourished, have foetal alcohol syndrome - even whether or not they are teething, etc. It also includes subtleties of speech, in and between questions, which hint at matters of medical concern. To an extent, taking account of these additional and often subtle contextual factors is important from the point of view of the study itself. Given that teething, malnutrition, and foetal alcohol syndrome, for instance, can affect the weight of the infant - and in a manner not necessarily related to TB - if the follow-up team can pick up on these details of context then there may be reason to spare the time-and-resource-consuming task of bringing the infant into the CV ward for extensive testing. Moreover, because TRO's current study is designed to determine the safety of a vaccine, it is even more relevant than in other studies to take stock of any problems and additional observations, no matter how apparently small or insignificant, which may be related to the trial vaccine.

The holistic and context-specific awareness required by the field teams' work demonstrates that the 'objectivity' of scientific practice is often grounded in a simultaneous need to remain sensitive to the contingencies of the everyday (Latour and Woolgar 1987; Mol 2006). However, another important reason for maintaining a critical, holistically trained eye throughout the follow-up visits, I was told, is that the home visits, and the intimacies they entail, yield occasions to give healthcare and support for infants that the mothers or relatives may not otherwise have access to or ask for. Although other study doctors and co-ordinators made very similar comments, Dr van Zyl advocated this approach most vocally:

What I was trying to implore them to do [in the TB Review] was to look beyond what they have to look for, because even though they're over to screen for TB and that sort of thing ... sometimes some of these kids might not get seen by someone with some sort of medical background ... If there are other things to identify, l'd like them to use it as an opportunity to screen for other things too - I'm sure Johan and Chrizane would agree as well ... It's so important that any time they have contact with anyone who has some insight into healthcare and links to resources that these opportunities get used as far as they possibly can.

With the current emphasis in policy and research on the role of the patient and the 'choices' they make as consumers of healthcare, it is easy to lose sight of the agencies of medical professionals in offering care where care is potentially needed (cf. Henderson 201 I:I78-9). The context sensitive approach to the challenges of healthcare provision urged by Annemarie Mol (2008), on the other hand, recommends that researchers maintain a holistic purview which acknowledges such agencies - even in the midst of bio-scientific experiments. Although this is by no means a general finding (see, eg., some 
of Petryna (2009) and Fischer's (2009) observations), there was a clear feeling on the part of TRO's study doctors that they had a responsibility for the wellbeing of their participants beyond the generic safeguarding required by study protocol.

Picking up on often subtle bodily and contextual signs is not easy in practice. As Yvonne, head of TRO's on-site quality assurance (QA) department, explained to me:

You're sending field workers with no medical
background into a situation to use an algorithm ...
or a questionnaire, yes/no, ask a question, get an
answer. The challenge is ... you ask the question,
and the human that's sitting in front of you in this
situation in the environment they are in, they see
this as an opportunity to discuss things, or to find
help, and they're dealing with someone who can't
help them, who doesn't have the insight to pick up
the hints or the medical information that is given in
those few sentences, for them to make an informed
decision and answer ... so I think they're struggling.

Whilst TRO's follow-up teams are currently undergoing training by the QA department to improve their holistic, context specific awareness when in the field, it is beyond the scope of TROs resources to send trained medical professionals into the field to conduct the follow-up visits. Hardon et al. (201 I:196) found that in under-resourced HIV testing facilities in East Africa, "the dynamics of care ... are shaped by the specificities of the care provided" and "professional training and human resource constraints." Unable to provide the 'ideal' degree of medical expertise, the field teams, in the midst of fulfilling the requirements of protocol (i.e. completing the "follow-up" form), nonetheless offer a unique, placespecific form of care for TRO's participants. Identifying the signs that 'home remedies' are not working, recommending visits to the local clinic, offering to drive them to the clinic and back again - these are but a few of the practices of care I witnessed the teams negotiating with the day-to-day requirements of protocol. For the most part, these practices are entirely ad hoc. As Yolanda put it, "when you leave the office you don't know what's going to happen, you only know when you get there." But they are nonetheless valuable contributions to TRO's 'extra-protocol' endeavours.

TRO's field researchers are also faced with another challenge, one that arises from the conjunction of an impoverished socio-economic environment and the intimacies necessitated by TB research: the requirement by the doctrines of Good Clinical Practice (GCP) - cemented in law by the Children's Act (2005) - to report cases of neglect and physical abuse. TRO's field teams are generally viewed as being in a position to notice such instances: being allowed into the infants' homes, interacting with mothers and relatives and, perhaps most revealing of all, seeing the infants naked. Unlike the bodily and contextual signs discussed above - many of which are framed as subtle, esoteric, requiring a 'trained' gaze - the latter, especially the phenomenon of physical abuse, are framed as relatively transparent, immediate, incumbent upon the field researcher's senses. Accordingly, TRO takes this issue very seriously. Yvonne argued passionately that protecting their research participants from instances of physical abuse is, and should continue to be, TRO's long-standing concern:

Now that you're entering that environment ... I mean this is something that we've always thought about ... [It's] impossible, actually, if you think about it, given what we know about our communities, not to know that kids are being abused, and what's going on in these homes.

However, during the time I spent with TRO's QA department and research staff at the field office, I became aware of a general perception that the follow-up teams encounter cases of severe neglect and abuse, but do not often report them, if at all. As Yvonne articulated the matter:

But we've never heard anything back from our field workers that, you know, where they've found instances of abuse ... very seldom would something like that come up. We think they see it, they hear about it, but they sort of ignore it because it's not part of their business, and they don't want to get involved.

In the days I spent out with one of the follow-up teams, I witnessed quite some perseverance on their part in probing these signs of abuse - often asking infants' mothers, as innocently as possible, about this cut or that bruise. This led me to question the extent to which the dichotomy which has been forged between the relative opacity of certain medically relevant signs and signals, and the relative transparency of abuse and, to a lesser extent, neglect - does justice to the ambiguities of what constitutes 'abuse' or 'neglect'. Certainly, the difference between 'neglect' and the inscription of apartheid legacy into the skins of TRO's participants is as thin as a hair's breadth. But even in the case of 'abuse' - which is arguably just as inseparable from the legacy of apartheid - it is not always a straightforward matter to distinguish a bruise left by the hand of an abusive father and one caused under more benign circumstances.

Nonetheless, a number of incidents involving TRO's enrolled infants - one involving a child being used as a human shield against an abusive husband - are cited as examples of the follow-up teams' failure to report more blatant cases of physical abuse. Rather than uncritically endorsing what is required by the Children's Act in these instances, however, the realities lived by TRO's field researchers invite us to challenge the notion that the requirements articulated by legal doctrines (such as the Act) are unambiguously 'good' or 'right' in all times and places. TRO's field teams have, over time, clearly earned a measure of trust with many constituents of the region, not only being allowed into their homes and their lives, but also to make physical contact with their children. These fragile relationships act as a medium through which healthcare can be provided, some of which goes beyond the requirements of research protocol. While this does not exempt TRO's follow-up teams from legal constraints, these are relationships which would be threatened were TRO's field workers known for 'telling' on infants' relatives. Moreover, some of the areas where TRO's participants 
live are incredibly dangerous. One of the follow-up teams recently witnessed a stabbing directly outside the TRO vehicle, after which the perpetrator held the knife up to the window and said, "I know who you are, and l'll get you next." Working in such environments, TRO's researchers have their own safety to consider. Thus, the requirements of the Children's Act (2005), whilst perhaps necessary, are not an indisputable 'good' enabling TRO's researchers to intervene in undesirable situations. Following Mol (2008), who highlights the efficacy of non-human as well as human actors, we should instead view legal doctrines such as the Children's Act as constituents of complex ecologies of care, the effects of which (see Mol 2006:4 12) vary from place to place and from time to time.

\section{The case verification ward}

One morning, I found myself regretting having accepted Monique's invitation to observe the goings-on in the CV ward's "treatment room", located down the hall, and through a set of swinging doors, from the ward itself. This was not only because of the early start it entailed. The infants, who had been admitted into the CV ward the previous day, were undergoing a procedure called "sputum induction", designed to procure a sputum sample which can be tested for TB disease. This is necessary because, unlike adults, infants are unable to cough up a sample on demand, and so the sputum, rather than being voluntarily coughed into a tube, has to be 'induced'. Whilst the nurses performing the inductions that morning, Monique and Klara, chatted amiably, intermittently alerting me as to what they were doing, I watched the procedure time and again with a mixture of fascination and anguish. After the infant has had the contents of their chests 'loosened' via the attachment of a nebuliser mask (assisted by the mother), the nurses would hold the infant down securely on the treatment table before lodging a thin tube, attached at its other end to a suction machine, up the infant's nose and down and into its throat. As soon as the tube is in place, sputum - and often a measure of blood - flies up the tube and into a test tube as the suction takes effect. The process was unpleasant to witness but it was of course far more of an ordeal for the infants, especially given that their mothers vacated the room as soon as the nebulising process was complete (see also Blake 2007).

Sputum induction is among the least pleasant of the highly structured regimen of tests and examinations the infants undergo during the 48 hours in the ward. Often the mothers or relatives simply fail to arrive with the enrolled infants, and it falls to the follow up visit teams to try and track them down again. However, in the cases of those who do attend the CV ward, rarely, if ever, is the protocol interrupted. On the day of admission, the majority of the study nurses' time is spent helping the mothers and infants to get settled into the ward. Each mother and infant is allotted an open cubicle with a bed and a cot and are given the time to meet the other mothers and infants with whom they will be sharing the next 48 hours. Such introductions and most of the subsequent interactions usually take place in the CV ward's large kitchen area or in front of the TV situated nearby. Throughout the rest of the day, the infants are given an HIV and Mantoux skin test and are taken for an X-ray in the radiology department. They are also weighed again. At about 4 o'clock on the morning of day two, after a four hour "starve", the infant has their first visit to the treatment room for a "gastric lavage". This has the same purpose as the sputum induction although it involves a slightly different method. Subsequently, and after a "milk feed", the infants are starved for another four hours before their second and final visit to the treatment room for the aforementioned sputum inductions. Immediately afterwards, and to conclude the most gruelling ordeals of the day, the infants undergo a quantiFERON blood test. Later that day, they are visited and examined by a clinical doctor, who, after checking through their case files to make sure nothing is amiss, signs the $\mathrm{CV}$ ward discharge form. The infants are discharged the next morning, and the mothers are allowed to take them home again, where they await a call or visit from TRO's researchers to alert them of the results of the tests and procedures of the stay.

Despite the unpleasantness of some requirements of the trial, and the inconvenience to the mothers of having to stay in the CV ward for 48 hours, there is the benefit of the close proximity to healthcare resources throughout. As Samsky (20II:4I2) notes, the line between experiment and care in the clinical trials industry is especially thin in instances where, as with TRO, the trials are conducted on the premises of larger medical facilities. Whereas the day-to-day operations of the follow-up teams (both protocol and extra-protocol) were inhibited by a lack of time and resources, in the CV ward medical expertise and resources were considerably more available. As in the hospital in the Netherlands where Mol (2008) conducted her ethnographic research, to my eye the healthcare relations enacted within the CV ward were comprehensive and largely heart-warming, although not free of challenges and conflict (cf. Greenhough 2010:137).

Given that there is a very real chance that some of TRO's enrolled infants will have potentially life-threatening TB disease, TRO cannot simply send an infant back home if a positive diagnosis is made. Treatment must be given. To that end, the close proximity of TRO's field office to the host hospital's clinical doctors and treatment facilities is crucial. The infants are visited by a clinical doctor (as opposed to an academic one) for clinical purposes. Occasionally, the clinical doctor is one of TRO's own, but mostly the host hospital's Dr Gilbert takes charge of the clinical component of TRO's infants' stays. Such are the consequences and worry of failing to pick up cases of TB disease that Dr Gilbert does not use TRO's trial algorithm to determine whether an infant is to be treated for TB disease. This is because childhood TB disease is so easy to 'miss', given the varying and often subtle signs and symptoms by which it manifests, that while 'accuracy' is the goal of the trial, in a clinical context doctors are more likely to err consciously towards over-diagnosis. As Dr Janssen remarked to me, "as a clinician, if you're worried, rather put them on TB treatment than miss it, and have them coming back with TB meningitis or something." Thus, the infants are subjected to two diagnostic algorithms during their stay in the CV ward: the trial algorithm for the study, and the more cautious Kibel system that $\mathrm{Dr}$ Gilbert uses to determine whether they should be treated for TB disease. ${ }^{3}$ Although positive diagnoses are in the minority and most infants are discharged from the $\mathrm{CV}$ ward after the normal 48 hours, infants who are 
diagnosed with TB disease are often admitted to the hospital's children's ward for a minimum of six months of extensive treatment. In particularly severe cases, infants are not even permitted to finish TRO's tests before being admitted into the children's ward. As Dr Gilbert divulged to me, one infant's lungs, recently, were in such bad condition that they were admitted to the hospital's children's ward almost immediately after being admitted to the CV ward. Thus, infants enrolled in TRO's trials are more likely than other infants in the surrounding rural region to be diagnosed and treated for one of the most prolific killers in South Africa today.

The CV ward's medical professionals seek to identify and manage other diseases as well as TB. Much like the follow-up teams' "holistic" awareness, the CV ward's nurses maintain a critical eye for medical matters that transcend the bare requirements of protocol. TRO's participants often arrive in the CV ward with ailments that require medical attention, or may develop them at some point throughout their 48 hour stays. As Monique explained to me, however, given that the primary purpose of the CV ward is the conduct of a bio-scientific experiment, which requires that 'variables' be tightly controlled (Latour 1991), managing these matters of medical concern is a complex affair. If, for instance, an infant arrives with signs and symptoms which do not point towards TB disease, they cannot be admitted to the CV ward. Rather, one of the nurses will refer the infant to one of TRO's doctors, who handles the case as if the infant had been referred to TRO's host hospital from one of the clinics in the surrounding communities. In cases where a child develops an ailment after admission, such as diarrhoea or a high temperature, the nurses still have to consult Dr Gilbert or one of TRO's study doctors, but efforts will usually be made to treat the problem within the CV ward until it appears that referral to the host hospital is the wiser course of action.

Notwithstanding the complexities of providing healthcare in the midst of an experiment, TRO's CV ward's links with more extensive healthcare resources allows earlier diagnoses and treatment than might otherwise be possible. Monique related one such example, reflecting also on the benefits of a visit to the $\mathrm{CV}$ ward:

One baby came in for failure to thrive. But when they [the nurses] did the physical exam, they could feel and see that around the abdomen there was some abnormality. They asked the study doc to do an assessment, and she found that there was a blockage, and they referred to the special paediatric outpatients. This baby ended up at Tygerberg hospital with colon problems. So ja, it's good for the infants: a free x-ray, HIV-test, quantiFERON, and we look at the overall health of babies.

Monique further told me that the possibility of earlier diagnosis and treatment is not restricted to the infants themselves, but also extended to the infants' mothers:

If a mum is coughing, you can see, we are all nurses, you can see someone's body language or facial expression when it's not well. So in informed consent, we'll ask if they're happy, you don't look satisfied, happy, are there any issues you'd like to discuss? They'll say, yes I'm worried about someone at home, I can't really stay for 2 days, or say I'm not feeling well, pain somewhere, I'm also coughing. So the nurse will ask Dr Gilbert or another doctor if we can do a chest x-ray, and they never refuse. The other night the night staff called to say it seems a mother might have breast cancer, a swollen painful breast ... So we let her call family to fetch her on the first evening ... She went to [another nearby] hospital. If they're coughing, or a skin rash, Dr Gilbert will also always try to prescribe something for them. That's the cool part.

Henderson (2011:65-6), on the basis of prolonged ethnographic encounters with people afflicted with AIDS, argues that the most valuable practices and relationships of care are enabled when those who are suffering can discuss things in their own time and on their own terms. One of the major successes of TRO's researchers in this regard (both in the CV ward and during the follow-up visits) has been to listen attentively to their participants and participants' relatives. While they could fulfil the requirements of protocol largely by 'ticking boxes' they often put the 'form' aside and acknowledge the human beings in front of them.

Yet, the relationship between experiment and care in the midst of TRO's endeavours is not complementary in all respects. Monique believed that, given that each enrolled infant is a part of the study for the first two years of their lives, some of the infants who come through the CV ward are old enough to remember the stay: "They're not babies anymore - they are old enough to remember, and they get affected." While the often unpleasant requirements of the CV ward visits might confer many benefits, Monique was concerned that some infants are admitted repeatedly to the CV ward when there is actually no need. She pointed out that one of the primary grounds for these infants being brought into the $\mathrm{CV}$ ward is their being underweight - which explains, at least, the frequency with which infants are weighed through their first years of life. However, she argued, whilst being underweight is one of the primary clinical signs of childhood TB, the fact that these infants are coming in repeatedly and found not to have TB - even by the more cautious clinical diagnostic mechanisms - is evidence that the weight loss is caused by other factors affecting their wellbeing. If they have foetal alcohol syndrome, for instance - a tragically common occurrence in South Africa - the infant is never going to be of a normal weight, "even if they live in the king's castle." More often, the underlying cause of being chronically underweight is simply under- or malnourishment.

Efforts have been made, however, to prevent infants returning to the CV ward for repeated stays. TRO cannot do much about infants with foetal alcohol syndrome besides acknowledging the condition when deciding whether to admit them to the $\mathrm{CV}$ ward. However, measures have been

3. This is named after Professor Maurice Kibel of the University of Cape Town. 
taken to improve the nutrition of underweight infants. As Monique recounted, the primary endeavour has been to put infants flagged as under- or malnourished on government feeding schemes, which are designed to ensure that they are connected to a consistent source of sustenance. Moreover, as was discussed above, efforts are being made to increase the follow-up visit teams' vigilance for factors of malnutrition that could cause infants to be losing weight or gaining it too slowly. Whilst largely ad hoc, and often unforeseen at the outset of the trial, these are instances of the kinds of "tinkering" that, according to Mol (2008:14), characterise the reality of caregiving relationships on the ground. The efficacy of these measures is certainly questionable: in addition to the issues discussed above, there is debate as to the extent to which infants actually receive the sustenance provided by the government feeding schemes. Yet, it is through tinkering with the resources available in the moment that TRO's researchers are able to negotiate, reconcile and ultimately improve both their protocol and 'extra-protocol' operations.

\section{Experiment and care: an uneasy union}

Latour (199I) has long argued that behind the 'objective' front often worn by scientific institutions can be found the complexities, place specificities and, perhaps most importantly, sociality inherent in their operations. A particular irony of the clinical trials industry is that, the more a researcher aims to accumulate data in the interests of such paradigmatic scientific values as reliability, validity and that most notorious one, objectivity, the more subjectivity the researcher encounters. The tensions inherent in this state of affairs as clinical trials 'go global' are the topic of a burgeoning body of literature in the social sciences. One of the most important observations made has been that 'Big Pharma', aided by the advent of CROs, is powerful enough to manipulate the global variability in regulatory standards to subject vulnerable people and populations to their prospective products (Petryna 2005, 2007, 2009). Yet, whilst gaping structural inequalities cast a long shadow over the conduct of clinical trials, current literature on practices of care (Mol 2006, 2008; Mol, Moser and Pols 2010; Henderson 20II; Hardon et al. 20II) recommends further ethnographic engagement with the particularities of diseases 'under the microscope', the local contexts and histories in which they are entwined, and how these inform the affective relationships between clinical research organisations and their participants. These are integral elements of the complex ecologies of care generated by clinical trials, and are thus important if we are to understand the challenges posed by the ground-level operations of the industry and to suggest meaningful solutions to those challenges.

My findings at TRO's field office suggest that the challenges its researchers faced in negotiating the day-to-day demands of protocol - and indeed 'extra-protocol' - are inseparable from the particularities of TB disease as a historical phenomenon in South Africa, the legacy of apartheid governance which has left the majority of TRO's participants in near-absolute poverty, and the prevailing political-economic conditions within which TRO operates. Fostering an holistic awareness for medical matters in the midst of poor living conditions; striking a balance between protocol and 'extraprotocol' with limited time and resources; negotiating legal requirements in difficult conditions; responding well when the requirements of protocol seem uncaring - these are some of the challenges that punctuate the ecology of care generated by TRO's clinical trials. They are specific to TRO and thus, in a sense, not generalisable. Yet, as Mol (2008:9) eloquently argues of her case study of practices of care in a hospital in the Netherlands:

This is not to say that its relevance is local. A case study is of wider interest as it becomes a part of a trajectory. It offers points of contrast, comparison or reference for other sites and situations. It does not tell us what to expect - or do - anywhere else, but it does suggest pertinent questions. Case studies increase our sensitivity. It is the very specificity of a meticulously studied case that allows us to unravel what remains the same and what changes from one situation to the next.

Although place-specific, the details of TRO's endeavours can serve as a point of comparison or reference for other localities of clinical research, especially those in resource-poor settings where interventions are arguably most urgently needed. What I would like to encourage is a greater collection of thick ethnographic accounts, the conversations between which will hopefully enhance our ability to suggest ground-level solutions to particular challenges in particular research localities.

I would like to offer one further suggestion for future ethnographic research on clinical trials. Mol (2008) advanced her arguments about the on-going processes of tinkering that characterise healthcare provision in the context of her engagement with chronic illness and the multiple actors involved therewith. TRO's endeavours suggest that 'tinkering', as a reality of providing and improving care, might well operate on larger scales too. As anthropologists, we are of course hesitant to read too much into notions such as 'community'. Yet, while the interactions between TRO's researchers and their participants are usually quite fleeting - at least compared to relations of chronic illness - these interactions with what are largely referred to by TRO's researchers as "the community" have spanned nearly 12 years. TRO's relationship with 'the community' has involved much tinkering over that period. It is at least partially in virtue of this that TRO is still generally trusted in and around the region. I suggest that the acknowledgement of tinkering manoeuvres at different scales might prove useful in further exploring the ins and outs of the clinical trials industry on the ground.

\section{References}

Adams, Vincanne et al. 2005. The Challenge of Cross-Cultural Clinical Trials Research: Case Report from the Tibetan Autonomous Region, People's Republic of China. Medical Anthropology Quarterly 19(3): 267-89.

Adams, Vincanne; Thomas Novotny, and Hannah Leslie. 2008. Global Health Diplomacy. Medical Anthropology 27(4): 315-323.

Benatar, Solomon. 2002. Reflections and Recommendations on Research Ethics in Developing Countries. Social Science and Medicine 54: ||3|-||4|.

Benatar, Solomon; Ross Upshur. 2010. Tuberculosis and Poverty: What can (and should) be Done? International Journal of Tuberculosis and Lung Disease 14(10): 1215-1221. 
Blake, Rosy. 2007. What a Cancer Diagnosis Can mean when you are a Child. Unpublished Manuscript. Social Anthropology Department, University of Cape Town.

Children's Act. 2005. (c.38: 12). Accessible at: http:// www.polity.org.za/attachment.php?aa_id= 2888. [Accessed: 05/ 06/I2].

Fischer, Jill. 2009. Medical Research for Hire: The Political Economy of Pharmaceutical Clinical Trials. New Brunswick, New Jersey: Rutgers University Press.

Greenhough, Beth. 2010. A logic of care beyond health geography. Area 42(I): 136-8.

Hardon, Anita; Emmy Kageha, John Kinsman, D. Kyaddondo, R. Wanyenze, and C.M. Obermeyer. 20II. Dynamics of Care, Situations of Choice: HIV Tests in Times of ART. Medical Anthropology 30(2): 183-20I.

Hatherill, Mark. et al. 2009. 'Structured Approaches for the Screening and Diagnosis of Childhood Tuberculosis in a High Prevalence Region of South Africa.' Bulletin of the World Health Organization. Accessible at: http://www.who.int/bulletin/volumes/ 88/6/09-062893.pdf [Accessed: 30/I I/I I].

Henderson, Patricia. 20I I. AIDS, Intimacy and Care in Rural KwaZuluNatal: A Kinship of Bones. Amsterdam: Amsterdam University Press.

Human, Oliver. 20II. The Rings around Jonathon's Eyes: HIV/AIDS Medicine at the Margins of Administration. Medical Anthropology 30(2): 222-40.

Latour, Bruno. 199I. We Have Never Been Modern, Trans. Catherine Porter, Brighton, UK: Harvester.

Latour, Bruno. 1999. Pandora's Hope: Essays on the Reality of Science Studies. Cambridge, MA: Harvard University Press.

Latour, Bruno and Stephen Woolgar. 1987. Laboratory Life: The Social Construction of Scientific Facts. Beverly Hills: Sage Publications.

Mol, Annemarie. 2006. Proving or Improving: On Health Care
Research as a Form of Self-Reflection. Qualitative Health Research 16(3): 405-I4.

Mol, Annemarie. 2008. The Logic of Care: Health and the Problem of Patient Choice. London: Routledge.

Mol, Annmarie, Ingunne Moser, and Jeannette Pols. 2010. 'Care: Putting practice into theory,' in Care in Practice. Edited by A. Mol, I. Moser, and J. Pols. pp. 7-27. Bielefeld: Transcript.

Naidoo, Pamela; Kelvin Mwaba. 2010. Helplessness, Depression and Social Support among People Being Treated for Tuberculosis in South Africa. Social Behaviour and Personality: An International Journal 38(10): 1323-1333.

Petryna, Adriana. 2005. Ethical Variability: Drug Development and Globalising Clinical Trials. American Ethnologist 32(2): 183-197.

Petryna, Adriana. 2007. Experimentality: On the Global Mobility and Regulation of Human Subjects Research. Political and Legal Anthropology Review 30(2): 288-304.

Petryna, Adriana. 2009. When Experiments Travel: Clinical Trials and the Global Search for Human Subjects. Princeton: Princeton University Press.

Rennie, Stuart. 2009. Review: When Experiments Travel: Clinical Trials and the Global Search for Human Subjects - by Adriana Petryna. Developing World Bioethics 10(2): II4-5.

Samsky. Martin. 201I. Review: Medical Research for Hire: The Political Economy of Pharmaceutical Clinical Trials, J. Fischer 2009. Medical Anthropology Quarterly 25(3): 4I 2-4.

Wallengren, Kristina et al. 20Il. Drug-Resistant Tuberculosis, KwaZulu-Natal, South Africa, 200I-2007. Emerging Infectious Diseases 17(10): 1913-6.

World Health Organization. 2009. 'WHO Report on the Tuberculosis Epidemic. Global Tuberculosis Control Reports.' [online] http://www.who.int/tb/publications/global_report/en/ [Accessed: 3/I2/II]. 